\title{
A Study on The Characteristics Of Miniature Floating Holographic Form And Expression-Focusing On Outdoor Floating Cylindrical Hologram -
}

\author{
Eun-Seo Song1, You-Suk Kim2, Sung-Dae Hong3*
}

\author{
${ }^{1}$ Reaserch Professor, Seokyeong Unversity, VR Future Center, 16-1 Jungneung-Dong Sungbuk-Ku Seoul, 136- \\ 704 Korea \\ ${ }^{2}$ Reaserch Professor, Seokyeong Unversity, VR Future Center, 16-1 Jungneung-Dong Sungbuk-Ku Seoul, 136- \\ 704 Korea \\ 3*Professor, Seokyeong Unversity, Dept. of Film and Digital Media, 16-1 Jungneung-Dong Sungbuk-Ku \\ Seoul, 136-704 Korea \\ songsun1999@gmail.com ${ }^{1}$, kimyousuk@gmail.com ${ }^{2}$, sungdaehong@gmail.com*3 \\ Corresponding author ${ }^{*}$ : mobile Phone: +82-010-5218-0267
}

Article History:Received:11 november 2020; Accepted: 27 December 2020; Published online: 5 April 2021

Abstract: Purpose of this study is to research the floating type cylindrical hologram that can also be embodied in the outdoor. This study identifies the floating hologram and its type, and researches the applied form design and realization type based on floating hologram realization type. For the manufacturing of miniature hologram, the floating mirror is installed in cylinder to embody new hologram type out of existing hologram. For the design of cylinder, the polarizing filter is used to minimize the diffracted reflection, and air intake device, the fan is installed to minimize the problem of heat and humidity. The study was able to check if realistic content that can be installed in outdoor space is embodied by researching how to minimize the problem of outdoor cylinder floating hologram.

Keywords: Hologram, Floating hologram, Holographic design, Oblique reflection, reflection, Miniature hologram

\section{Introduction}

A hologram is a physical recording of an interference pattern which uses diffraction to reproduce a threedimensional light field, resulting in an image which retains the depth, parallax, and other properties of the original scene.[1] The hologram can provide realistic information to viewers by providing three-dimensional virtual images to the real world. Nowadays, the most beautiful 3D pictures and movies are created by means of holograms.[2] The commonly thought 'hologram' is a hollow video that literally refers to a video floating in the air. However, such stereoscopic imaging technologies that appear in SF have yet to be developed. Of course, considering that the fundamental principle of empty images is the interference of waves of light, it is not impossible, but possible in reality.[3] The people-centered visuals account for $80 \%$ of hologram, "virtual world + real world" is super-converged, which allows people can embody the spatial data in front of the eyes super realistically [4] and the hologram is applied and researched in various fields through convergence with leading technologies of the fourth industrial revolution. According to changes in hologram technology, a variety of holograms are being introduced, ranging from previous analog hologram to now AI applied hologram.[5] The structure and form of hologram are also changing in accordance with technology changes. This requires continuous study of the various forms of hologram and the types of realization according to shape deformation. This study identifies floating hologram and its type and researches the form design and realization type applied based on floating hologram realization type.

\section{Floating hologram classification}

\subsection{Floating hologram}

The term 'hologram' is a combination of 'holo', which means perfect or whole, and 'gram', which means message or information. [6] It is produced based on a technology called "Pepper's Ghost," in this technology, Henry Pepper, who was a theater director in the 1860s, installed a 45-degree angle glass plate on the stage and projected a lantern to make a ghost figure appear on the stage. Floating hologram is a technology that installs transparent mirrors at an angle of 45 degrees and produces images that appear to be floating in the air as image contents are reflected or transmitted in transparent mirrors. [Figure 1, 2] 


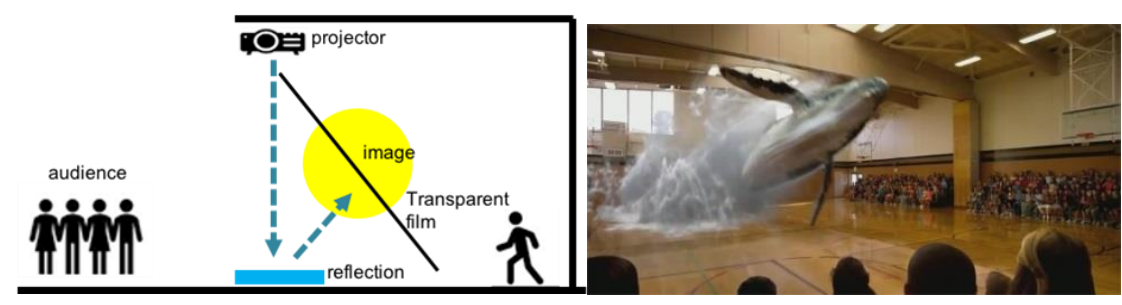

Figure 1. Floating hologram

Figure 2. The promotion using holography technology of Google

\subsection{Classification of small floating hologram}

The floating hologram of the display device can be embodied as miniature holograms, which are classified into reflective hologram, multi-layer hologram, multi-angle hologram. The floating type is a section hologram method that can express live or video contents as if they are floating in the air, and mainly uses floating mirrors or transparent films. Figure 3 is a hologram content that combines floating type hologram and AR game

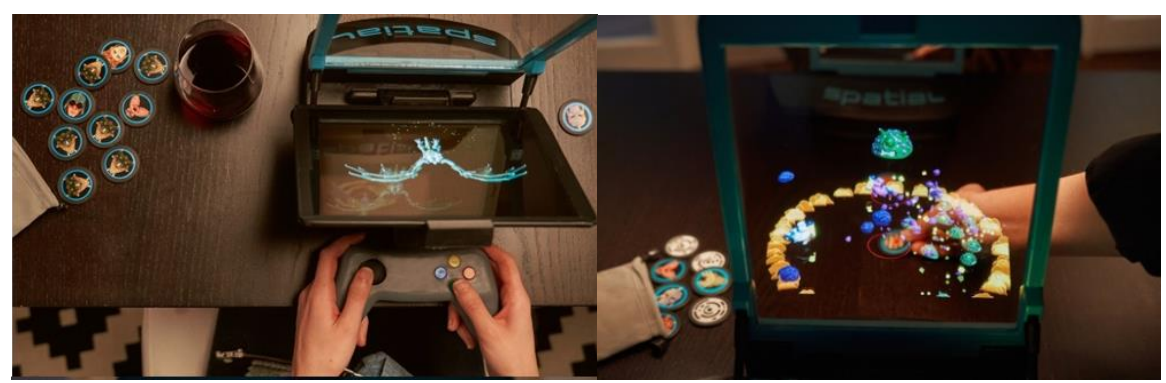

Figure 3. AR Game 'Spatial' using floating hologram (spatial)

Figure 2 shows the interactive special effects for users of night forest parks. In order, it is Multi-layer hologram type is a hologram type that consists of two or more floating mirrors. Each floating mirror has a projector or an image expression panel to create a sense of depth by making two or more reflected images.

\section{Figure4. Multi-layers type (I3DG Palm top theater)}

The multi-angle hologram type is a hologram that consists of two or more faces created by a floating mirror. Unlike multiple layers, it embodies three-dimensional effect, not a sense of depth.

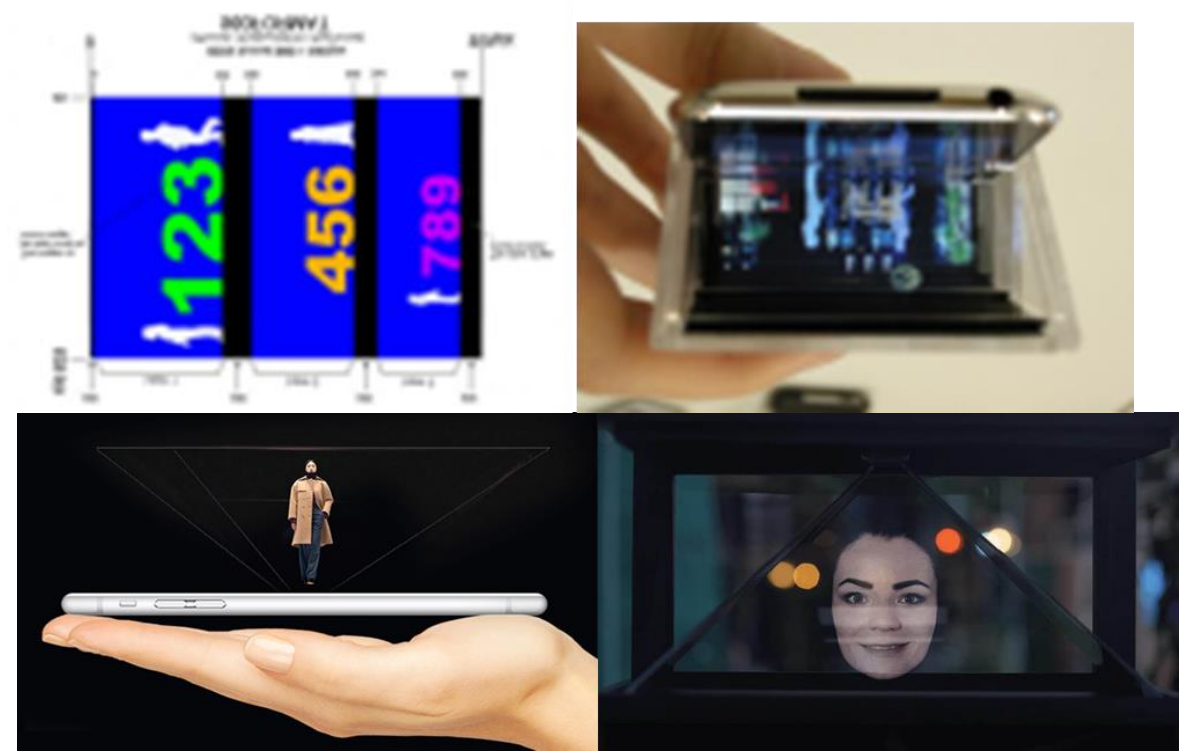

Figure5. A fashion show that embodies the 3D hologram principle using a polyhedral reflector with a mobile phone (The studio $k$ )

3. Outdoor floating cylindrical hologram device
3.1. Design of outdoor floating cylindrical hologram 
The hologram realization type currently commercialized consists of the hologram that uses multiple faces and single face. The floating cylindrical hologram type is basically composed of floating type hologram. The principle of floating hologram type is to project the video contents embodied through a single expression panel on the half mirror making the video contents appear to be floating in the empty space of half mirror.[7] [Figure 6] In cylindrical hologram, elliptical hologram half mirror is installed at an angle of 45 degrees inside the transparent cylinder and LCD panel is installed at the top of the cylinder, making the video contents appear to be floating in the cylinder. It is common for hologram to ensure that the video content projected from the LCD panel does not appear directly to the viewer's view, making it appear to be floating in the image only in the half mirror. Rather than installing panels at the bottom, the panel was installed at the top to design the video contents appear to be floating in the air. It intends to express a high sense of immersion and realism by using cylindrical form out of the structure of the existing floating type, resulting in the hologram video embodied in the cylinder allows the viewers to access to the hologram images that are transparent in all aspects.

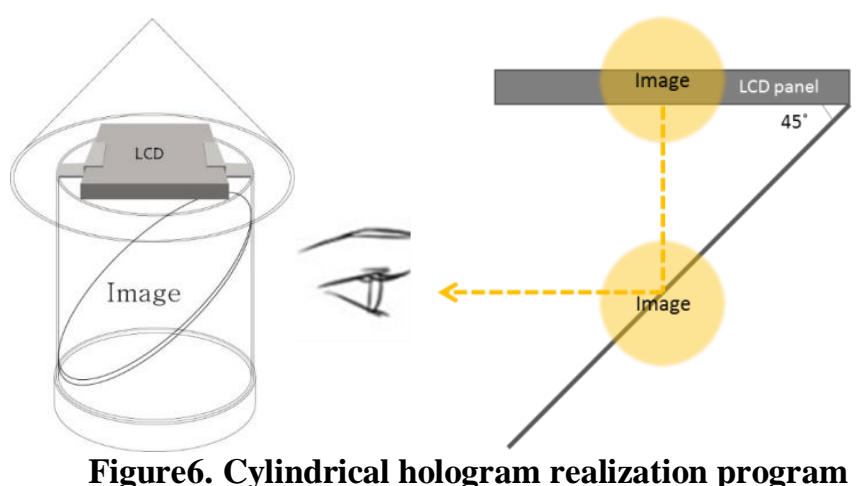

For the structure installed outdoor, water proof and dust resistance should be considered. For structures installed in the outdoor, it should be designed by considering waterproof and dustproof. Cylindrical hologram is more likely to become damp inside the cylinder in case of rain. [Figure 7] is designed to block moisture inside the cylinder, 8 fans are installed on top of the cylinder hologram to minimize heat from the LCD panel, and air intake devices is installed on the bottom to vacuum the inside of the cylinder to minimize moisture even if installed in the outdoor.

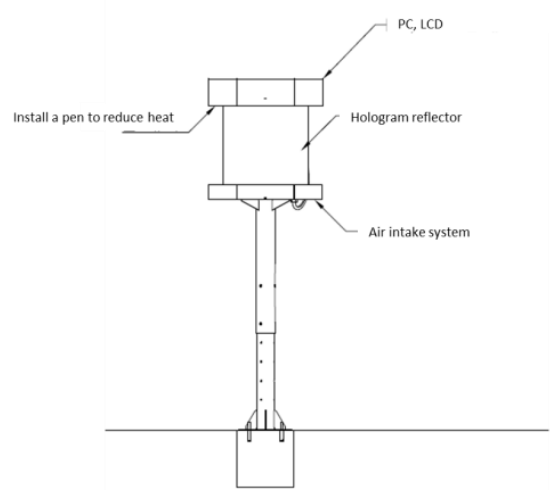

Figure 7. Design of cylindrical hologram

In order to fully watch the hologram, the floating mirror installed on the top should be horizontal with the viewer's eye level for optimized hologram view.[8] Otherwise, it is difficult to see the exact image of the video content if the floating mirror is much higher or lower than the viewer's eye level. In order to watch the hologram without image deformation, viewers can watch the hologram video regardless of viewing distance when the viewer and the floating mirror are horizontal. Unlike indoor environments, outdoor environments have the height of ground. In order to install hologram on the outdoor, the viewer's eye level and the ground height should be considered. [Figure 8] is designed to adjust the height of the hologram support from a minimum of $800 \mathrm{~mm}$ to a maximum of $1400 \mathrm{~mm}$. 


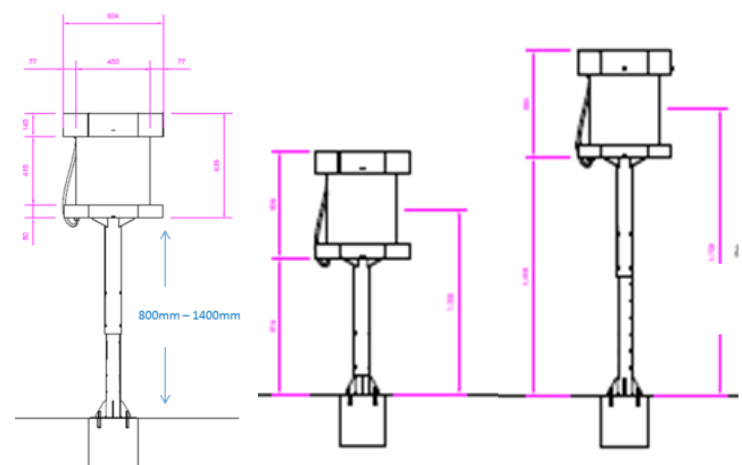

Figure8. Cylindrical hologram height adjustment range

\subsection{Outdoor floating cylindrical hologram diffracted reflection phenomenon}

\subsubsection{Polarizing filter}

Basically, the light oscillates in all directions on a line at right angles. When a polarizing filter is used, it makes light oscillate in one direction. Therefore, if the polarizing filter is installed in the path of light, the oscillation of almost light blocks the filter, resulting in light waves in one direction. [Figure 9]

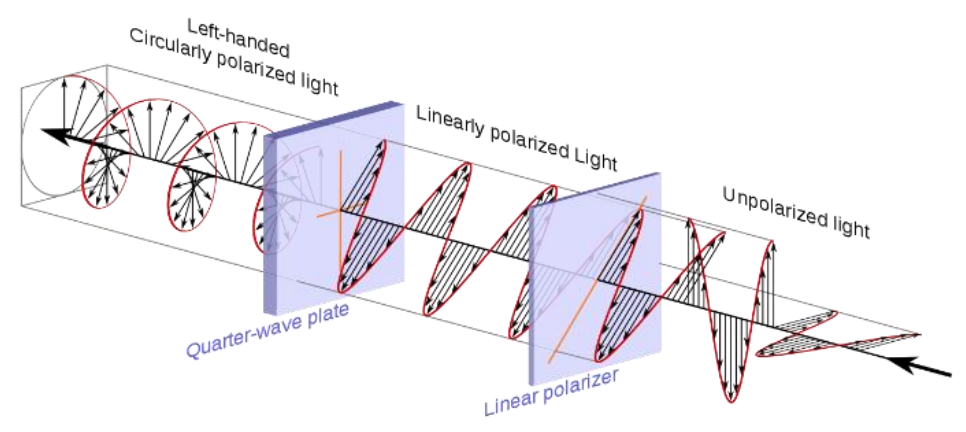

Figure9. Polarizing filter[10]

\subsubsection{Polarizing filter}

The cylindrical hologram is a video contents projecting LCD in which the elliptical floating mirror is installed in the transparent acryl cylinder at 45 degree and attached on its top. LCD light is projected at 178 angle and if there is no reflected face, the image is created in the floating mirror only. In cylindrical hologram, LCD light is not projected on the floating mirror only but diffracted and reflected in the inner wall of cylinder, making the projected image is also created in the transparent acryl cylinder, resulting in the viewers will be less immersed in watching the hologram video.
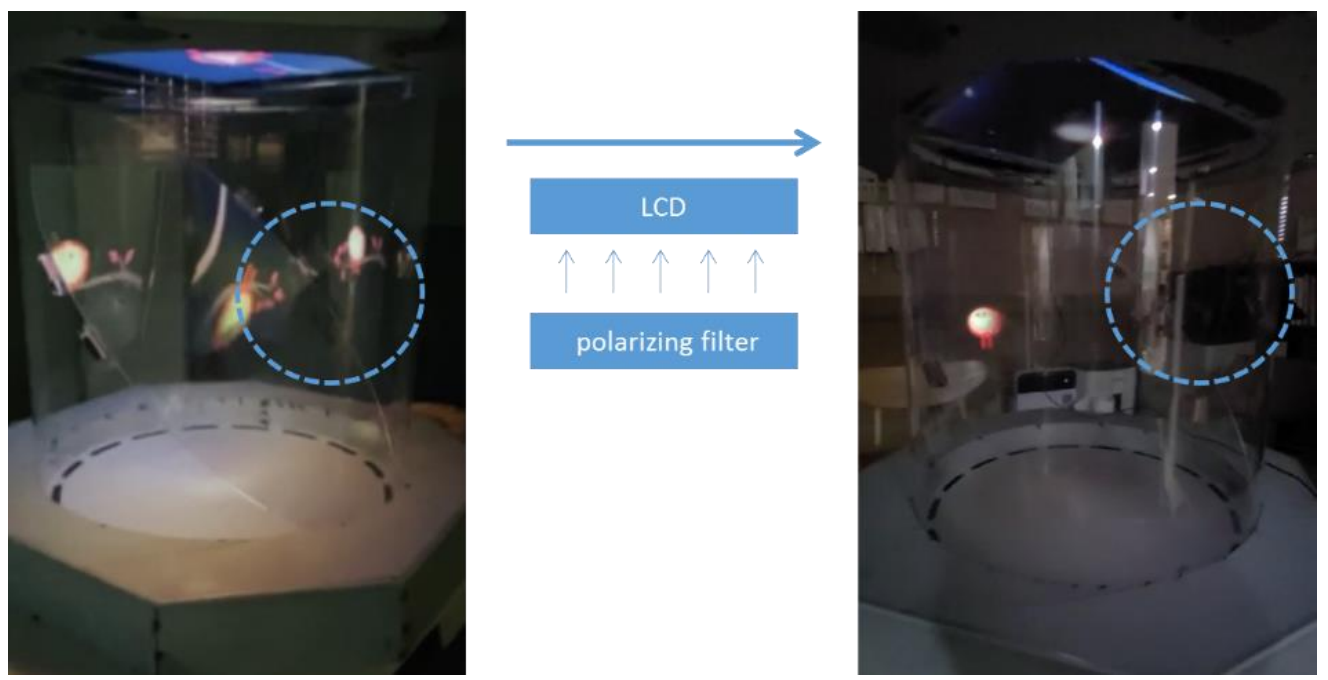

Figure10. Improvement of hologram diffracted reflection phenomenon

The diffracted and reflected light can be projected in one direction by attaching the polarizing filter above mentioned on LCD panel. [Figure 10] minimizes the diffracted and reflected hologram image by attaching the 
polarizing filter. As a result, the diffracted reflection is minimized but the projected image gets darker.

\subsubsection{Outdoor floating cylindrical hologram form design}

The outdoor floating cylindrical hologram will be installed at Nammangsan Sculpture Park in Tongyeong, Korea. In order not to harm the landscape of the park, the wood material was chosen and designed in the form of a hut to express the house where small fairies live. [Figure 11]

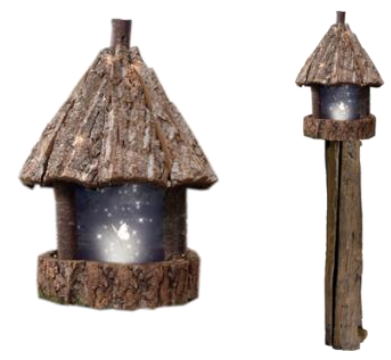

Figure11. Hologram form design

\section{Conclusion}

Currently, realistic content of the 4th industry is being conducted based on virtual reality (VR), augmented reality (AR), and mixed reality (MR), and research on miniaturized devices is continuing. Through such research, the accessibility of users to the technology of realistic content is maximized. As holograms gradually change from large size to small size, its accessibility is expanding more and more. This paper suggests the design of the hologram, which was mainly used for performances, by miniaturizing it so that the viewers can view hologram contents even in a narrow space for the manufacturing of miniature hologram, the floating mirror is installed in cylinder out of existing hologram type to embody new hologram type. For the design of cylinder, the polarizing filter is used to minimize the diffracted reflection of light, and air intake device is used for the installation in outdoor space and the fan is installed to minimize the problem of heat and humidity. The study was able to check if realistic content that can be installed in outdoor space was embodied by researching how to minimize the problem of outdoor cylinder floating hologram. The hologram belongs to the realistic content and is under continuous research. However, miniaturized hologram, outdoor hologram, and various types of hologram research should be continuously conducted.

\section{Acknowledgements}

This research is supported by Ministry of Culture Sports and Tourism(MCST) and Korea Creative Content Agency(KOCCA) in the Culture Technology(CT) Research \& Development Program 2020.

\section{References}

1. Information on http://holocenter.org/

2. Adeleh Granmayeh Rad, Hamed Abbasi, Talie Zarei. A Study on Advances in Creating 3D Holographic Images and Optical Applications of Holography. The International Conference on INnovation and Collaboration in Engineering Research Second Edition. 2013 June 20-21.

3. Information on

4. https://namu.wiki/w/\%ED\%99\%80\%EB\%A1\%9C\%EA\%B7\%B8\%EB\%9E\%A8

5. Information on http://www.iminju.net/news/articleView.html?idxno=33235

6. Information on http://www.iminju.net/news/articleView.html?idxno=33235

7. Kyoo-jin Oh,Soon-kak Kwon. Real-time Implementation of Character Movement by Floating Hologram based on Depth Video. 2017 Dec:4(4):289-294.

8. Jang-won Park, Tai-wan Kim. Implementing Video Content on Top-Down Floating Hologram Device. Korea society of design trend. 2018 Jan: 58(1): 67-78.

9. Jang-won Park, Tai-wan Kim. Implementing Video Content on Top-Down Floating Hologram Device. Korea society of design trend. 2018 Jan: 58(1): 67-78.

10. Information on https://en.wikipedia.org/wiki/Polarizing_filter_(photography)

11. Information on https://en.wikipedia.org/wiki/Polarizing_filter_(photography) 